\title{
RELATIONS ENTRE LA COMPOSITION DU PLASMA SANGUIN ET LES SYMPTÔMES DE TÉTANIE D'HERBAGE CHEZ LES BOVINS
}

\author{
P. LARVOR \\ avec la collaboration technique de Nicole Berthelot et Yvonne Tandeau de Marsac \\ Service de Nutrition minérale, École nationale vétérinaire, Alfort (Seine).
}

SOMMAIRE

Les relations entre la composition du plasma sanguin (pour le calcium, le phosphore total, le magnésium, le sodium, le potassium, le $\mathrm{pH}$ et les protéines) et les symptômes de Tétanie d'herbage des bovins, notés arbitrairement de o à 5 , ont été étudiées sur 43 échantillons de plasma provenant de $\mathrm{I} 2$ vaches saines et de $3 \mathrm{I}$ vaches à différents stades de la maladie. Seule la diminution du magnésium et du calcium plasmatiques est susceptible d'expliquer une fraction appréciable de la variance des symptômes : la corrélation multiple qui relie le magnésium et le calcium plasmatiques aux symptômes de Tétanie d'herbage est $\mathrm{R}=0,703$ (limite de $\mathrm{R}$ pour $\mathrm{P}<0, \mathrm{OI}=0,494$ ). Compte tenu des nombreuses sources d'erreur, il semble donc possible que les variations du magnésium et du calcium plasmatiques puissent expliquer la totalité des symptômes observés.

On a par ailleurs constaté une relation positive entre le $\mathrm{pH}$ du plasma et la pression atmosphérique $(r=+0,359, \mathrm{P}<0,05)$ et une relation positive entre le magnésium plasmatique et le nombre de jours écoulés depuis le vêlage $(r=+0,506, \mathrm{P}<0,01)$.

La découverte de 1'hypomagnésiémie dans la Tétanie d'herbage, par SJollEma et SEEKLES (I930), n'a pas pour autant résolu le problème du déterminisme de la tétanisation musculaire observée lors de cette maladie.

On devait rapidement s'apercevoir qu'un magnésium sérique faible ne s'accompagnait pas forcément de symptômes cliniques, et que des symptômes graves étaient parfois associés à une baisse du magnésium relativement modeste (voir tableau 3).

Les réactions à cette constation furent de deux types : les uns (biochimistes principalement) ne considérèrent comme Tétanie d'herbage que les cas où l'hypomagnésiémie était manifeste ; les autres (cliniciens principalement) considérèrent comme Tétanie d'herbage tout syndrome d'allure tétanique survenant à la mise au pâturage. Les uns ont tendance à prendre le symptôme biochimique pour la maladie, et, si objectif et si quantitatif que soit un symptôme biochimique, il est imprudent de le considérer a priori comme pathognomonique; quant aux autres, leur attitude les 
TABLE

Résultats des analyses plasmatiques d'animaux sains ou à différents

\begin{tabular}{|c|c|c|c|c|c|}
\hline \multirow{2}{*}{ No de l'animal } & \multirow{2}{*}{ Nombre de veaux } & Stade de lactation & Race & Symptômes & \multirow{2}{*}{ pH plasma } \\
\hline & & nombre de jours & $\begin{array}{c}\mathrm{F}=\text { flamande } \\
\mathrm{H}=\mathrm{F} . \mathrm{F} . \mathrm{P} . \mathrm{N}\end{array}$ & (Échelle arbitraire) & \\
\hline$A_{1} \ldots \ldots \ldots \ldots \ldots$ & 7 & 40 & $\mathrm{~F}$ & 3,0 & 7,68 \\
\hline$A_{2} \ldots \ldots \ldots \ldots \ldots$ & 3 & 21 & $\mathrm{~F}$ & 3,5 & 7,84 \\
\hline $\mathrm{B}_{1} \ldots \ldots \ldots \ldots \ldots \ldots$ & 4 & 60 & $\mathrm{~F}$ & 3,0 & 7,70 \\
\hline$B_{2} \ldots \ldots \ldots \ldots \ldots \ldots$ & 3 & 21 & $\mathrm{~F}$ & 1,0 & 7,72 \\
\hline $\mathrm{B}_{2}$ bis $\ldots \ldots \ldots \ldots$ & - & - & -- & 4,0 & 7,68 \\
\hline $\mathrm{B}_{2} \operatorname{ter} \ldots \ldots \ldots \ldots$ & - & - & $\cdots$ & 0,0 & 7,62 \\
\hline $\mathrm{C}_{1} \ldots \ldots \ldots \ldots$ & 4 & 90 & $\mathrm{~F}$ & 1,0 & 7,60 \\
\hline $\mathrm{D}_{1} \ldots \ldots \ldots \ldots \ldots$ & 4 & 90 & $\mathrm{~F}$ & 2,5 & 7,65 \\
\hline $\mathrm{D}_{2} \ldots \ldots \ldots \ldots \ldots$ & 3 & 180 & $\mathrm{~F}$ & 0,0 & 7,61 \\
\hline $\mathrm{D}_{3} \ldots \ldots \ldots \ldots \ldots$ & 4 & 90 & $\mathrm{~F}$ & 0,0 & 7,58 \\
\hline ............. & 1 & 15 & $\mathrm{~F}$ & 3,5 & 7,80 \\
\hline $\mathrm{F}_{1} \ldots \ldots \ldots \ldots \ldots$ & 5 & 85 & $\mathrm{~F}$ & 1,0 & 7,70 \\
\hline $\mathrm{G}_{1}, \ldots \ldots \ldots \ldots \ldots$ & 3 & 90 & $\mathrm{~F}$ & 3,0 & 7,74 \\
\hline $\mathrm{H}_{1} \ldots \ldots \ldots \ldots \ldots$ & 4 & 58 & $\mathrm{~F}$ & 4,5 & 7,48 \\
\hline $\mathrm{J}_{1} \ldots \ldots \ldots \ldots \ldots \ldots$ & 5 & 150 & $\mathrm{~F}$ & 0,0 & 7,74 \\
\hline $\mathrm{J}_{2} \ldots \ldots \ldots \ldots \ldots \ldots$ & $?$ & $\vdots$ & $\mathrm{F}$ & 0,0 & 7,68 \\
\hline n........ & 1 & 180 & $\mathrm{H}$ & 1,5 & 7,65 \\
\hline $\mathrm{K}_{2} \ldots \ldots \ldots \ldots \ldots \ldots$ & 4 & 30 & $\mathrm{H}$ & 2,5 & 7,73 \\
\hline $\mathrm{L}_{1}$ & 7 & 120 & $\mathrm{~F}$ & 0,0 & 7,65 \\
\hline $\mathrm{I}_{2} \ldots \ldots \ldots \ldots \ldots$ & 1 & 90 & $\mathrm{~F}$ & 0,0 & 7,67 \\
\hline$M_{1}$ & 4 & 30 & F & 4,0 & 7,69 \\
\hline $\mathrm{N}_{2}$ & 4 & 40 & $\mathbf{F}$ & 3,0 & 7,64 \\
\hline$O_{1}^{*}$ & 2 & 63 & $\mathrm{H}$ & 1,0 & 7,66 \\
\hline $\mathrm{P}_{1}$. & 4 & 74 & $\mathbf{F}$ & 0,0 & 7,68 \\
\hline $\mathrm{P}_{2} \ldots \ldots \ldots \ldots \ldots \ldots$ & 3 & ? & $\mathrm{F}$ & 0,0 & 7,65 \\
\hline$Q_{1}, \ldots \ldots \ldots \ldots \ldots$ & 2 & 90 & F & 4,0 & 7,67 \\
\hline $\mathrm{R}_{1} \ldots \ldots \ldots \ldots \ldots$ & 3 & 210 & $\mathrm{~F}$ & 4,0 & 7,77 \\
\hline $\mathrm{S}_{1} \ldots \ldots \ldots \ldots \ldots$ & 1 & 24 & $\mathrm{~F}$ & 4,0 & 7,82 \\
\hline $\mathrm{S}_{2} \ldots \ldots \ldots \ldots \ldots$ & 1 & 71 & $\mathrm{~F}$ & 0,0 & 7,80 \\
\hline $\mathrm{S}_{3} \ldots \ldots \ldots \ldots \ldots$ & 1 & 72 & $\mathrm{~F}$ & 0,0 & 7,75 \\
\hline $\mathrm{T}_{1} \ldots \ldots \ldots \ldots \ldots \ldots$ & 5 & 150 & $\mathrm{~F}$ & 1,0 & 7,72 \\
\hline $\mathrm{T}_{2}, \ldots \ldots \ldots \ldots$ & $?$ & $?$ & $\mathrm{~F}$ & 1,0 & 7,76 \\
\hline $\mathrm{T}_{3}^{2} \ldots \ldots \ldots \ldots \ldots$ & $?$ & $?$ & $\mathrm{~F}$ & 1,0 & 7,70 \\
\hline$U_{1} \ldots \ldots \ldots \ldots$ & 2 & 45 & $\mathrm{~F}$ & 2,0 & 7,73 \\
\hline$V_{1} \ldots \ldots \ldots \ldots \ldots$ & 3 & 150 & $\mathrm{H}$ & 2,0 & 7,78 \\
\hline $\mathrm{w}_{1} \ldots \ldots \ldots \ldots$ & 8 & 42 & $\mathrm{~F}$ & 1,0 & 7,70 \\
\hline $\mathrm{x}_{1} \ldots \ldots \ldots \ldots \ldots$ & 1 & 180 & $\mathrm{H}$ & 1,5 & 7,70 \\
\hline$Y_{1} \ldots$ & 5 & 90 & $\mathrm{~F}$ & 3,0 & 7,74 \\
\hline (1) & 4 & 150 & $\mathrm{~F}$ & 1,5 & 7,60 \\
\hline 1 & 2 & 150 & F & $0, \overline{3}$ & 7,60 \\
\hline$x_{2}$ & 2 & 210 & $\mathrm{~F}$ & 0,0 & 7,62 \\
\hline & 3 & 60 & $\mathrm{H}$ & 3,5 & 7,59 \\
\hline , & 6 & 60 & $\mathbf{F}$ & 1,0 & 7,66 \\
\hline
\end{tabular}


$\mathbf{U} \mathbf{I}$

tades de la maladie (Échelle arbitraire des symplômes de 0 à 5 ) lans la tétanie d'herbage

\begin{tabular}{|c|c|c|c|c|c|}
\hline K plasma & Na plasma & Ca plasina & P total plasma & Mg plasma & $\begin{array}{c}\text { Protéines du } \\
\text { plasma }\end{array}$ \\
\hline pour 1000 & pour 1000 & $\mathrm{mg} / 100 \mathrm{ml}$ & $\mathrm{mg} / 100 \mathrm{ml}$ & $\mathrm{mg} / 100 \mathrm{ml}$ & (jour 100) \\
\hline $0,2: 37$ & $3, \geq 1$ & 05,9 & 08,2 & $0,6:$ & 08,75 \\
\hline 0,218 & 2,18 & $0_{t, 0}^{\prime}$ & 06,2 & 0,41 & 08,85 \\
\hline $0,28^{\prime}$ & 2,18 & $0_{4}^{\prime}, t_{t}^{\prime}$ & 10,6 & 0,47 & 08,90 \\
\hline 0,163 & 3,10 & 08,3 & $12, k$ & 0,56 & 08,90 \\
\hline 0,203 & 3,2 & $0 \div, 9$ & 12,7 & 0,48 & 10,20 \\
\hline 0,141 & 3,22 & 10,5 & 12,7 & 1,26 & 08,30 \\
\hline $0,16^{\prime} k$ & 3,20 & 10,3 & 12,4 & 0,62 & 07,20 \\
\hline 0,188 & 3,07 & $08, \bar{i}$ & 10,3 & 0,57 & 08,80 \\
\hline $0,15:$ & 3,10 & 10,2 & 10,0 & 1,22 & 08,05 \\
\hline 0,173 & 3,04 & 10,0 & 10,9 & 0,78 & 08,70 \\
\hline 0,180 & 3,16 & 10,1 & 08,0 & 0,49 & 06,60 \\
\hline 0,195 & 3,13 & 07,8 & 09,4 & 0,40 & 08,75 \\
\hline 0,160 & 3,19 & 08,5 & 13,8 & 0,45 & 07,15 \\
\hline 0,153 & 3,12 & 04,3 & 10,9 & 0,50 & 08,80 \\
\hline 0,182 & 3,19 & 09,0 & 10,6 & 0,89 & 07,05 \\
\hline 0,165 & 3,16 & 10,4 & 10,6 & 0,57 & 07,20 \\
\hline 0,147 & 3,21 & 09,5 & 14,7 & 0,77 & 07,75 \\
\hline 0,140 & 3,21 & 09,7 & 13,6 & 0,68 & 07,75 \\
\hline 0,143 & 3,02 & 10,5 & 10,0 & 2,06 & 07,80 \\
\hline 0,146 & 2,98 & 10,0 & 09,7 & 2,07 & 07,00 \\
\hline 0,141 & 2,91 & 05,1 & 08,8 & 1,03 & 08,30 \\
\hline 0,203 & 3,11 & 07,7 & 12,4 & 0,66 & 06,95 \\
\hline 0,159 & 3,07 & 09,6 & 15,0 & 1,10 & 08,75 \\
\hline 0,150 & 2,95 & 09,6 & 09,4 & 1,96 & 07,15 \\
\hline 0,153 & 2,98 & 09,4 & 09,7 & 1,60 & 07,15 \\
\hline 0,168 & 3,00 & 05,4 & 07,7 & 0,79 & 07,50 \\
\hline 0,211 & 3,07 & 06,8 & 15,3 & 0,51 & 07,30 \\
\hline 0,261 & 3,10 & 06,7 & 11,8 & 0,43 & 06,50 \\
\hline 0,234 & 3,09 & 07,5 & 15,0 & 0,37 & 07,10 \\
\hline 0,183 & 3,49 & 10,0 & 11,2 & 2,09 & 07,00 \\
\hline 0,139 & 3,06 & 04,1 & 03,8 & 0,73 & 07,35 \\
\hline 0,203 & 3,12 & 09,0 & 08,8 & 0,18 & 08,00 \\
\hline 0,162 & 2,97 & 09,4 & 10,0 & 0,93 & 08,20 \\
\hline 0,169 & 3,07 & 10,1 & 11,1 & 0,56 & 08,30 \\
\hline 0,187 & 2,96 & 09,3 & 08,8 & 1,13 & 09,20 \\
\hline 0,172 & 2,89 & 08,5 & 08,8 & 1,06 & 09,35 \\
\hline 0,206 & 3,22 & 09,1 & 11,2 & 1,94 & 08,85 \\
\hline 0,188 & $3,1_{t}^{\prime}$ & 10,4 & 12,4 & 0,69 & $08, \mathbf{1 0}$ \\
\hline 0,171 & 2,97 & 07,3 & 07,4 & 2,10 & 07,35 \\
\hline 0,177 & 3,05 & 10,2 & 13,0 & 1,53 & 07,60 \\
\hline 0,152 & 3,10 & 09,7 & 14,1 & 2,07 & 08,20 \\
\hline 0,221 & 3,18 & 04,6 & 08,5 & 0,60 & 08,60 \\
\hline 0,170 & 3,05 & 07,0 & 10,0 & 1,65 & 08,90 \\
\hline
\end{tabular}


expose à prendre pour argent comptant tous les diagnostics téméraires, tous les prélèvements non représentatifs et tous les dosages incertains.

La méthodologie d'une étude des relations symptômes-biochimie est donc rendue difficile, soi parce qu'on risque de choisir les animaux malades à étudier sur des critères biochimiques, donc de présupposer la réponse avant d'avoir posé la question, soit parce qu'on risque d'englober des syndromes différents, de symptomatologie voisine. Nous pensons que le meilleur moyen d'obtenir un résultat valable est de sélectionner les animaux à étudier sur des critères symptomatologiques, mais en étant extrêmement strict quant au diagnostic, et en éliminant tous les cas un tant soit peu douteux, fût-ce au risque d'écarter de l'étude des cas authentiques. Ainsi un animal trouvé agonisant le matin au pâturage peut être victime de Tétanie d'herbage, mais bien souvent il est dans un tel état d'épuisement musculaire que la tétanisation n'est pas très apparente. Quelle que soit la vraisemblance du diagnostic on ne peut l'inclure dans une étude qui ne doit rassembler que des cas certains.

\section{MATÉRIEL ET MÉTHODES}

La maladie a été étudiée entre le ıo et le 25 mai ig6r dans sa zone de fréquence la plus élevée en France (Larvor, Brochart et Théret, I96i) autour de Hazebrouck, Bergues et Cassel (Nord), en collaboration avec les vétérinaires de ces localités.

Le diagnostic étant posé, le sang était prélevé avant tout traitement dans un tube hépariné, centrifugé aussi rapidement que possible (quelques heures) pour l'isolement du plasma. Le pH du plasma, était mesuré immédiatement ( $\mathrm{pH}$ mètre Biolyon), et le plasma, additionné de merth:olate, conservé en chambre froide pour des analyses ultérieures. Nous avons ainsi récolté 43 échantillons de plasma, dont $\mathbf{I} 2$ de vaches saines et $3 \mathbf{I}$ de vacher ì différents stades de la maladie.

Les détails concernant chacun de ces animaux nombre de veaux, stade de lactation, race, gravité des symptômes) sont consignés dans le tablaau $\mathrm{I}$, avec les résultats des analyses plasmatiques. Chaque lattre correspond à une exploitation, chaque numéro suivant la lettre à un animal de l'exploitation ; les numéros $\mathrm{B}_{2}, \mathrm{~B}_{2}$ bis et $\mathrm{B}_{2}$ ter désignent un même animal à trois stades de l'évolution de la maladie. tation.

Le tableau 2 donne les principaux renseignements concernant les pâturages de chaque exploi-

Les symptômes étaient notés de o à 5 en employant l'échelle arbitraire suivante :

$\circ$ : Aucun symptôme.

I : Baisse de la production laitière, nervosité, comportement anormal (animal à l'écart du troupeau).

2 : Boiterie, raideur, léger tremblement des muscles de l'épaule ou de la croupe.

3 : Difficulté de locomotion nette, chancellement, tremblement accentué, trismus.

4 : Crise de Tétanie typique.

5 : Crise entraînant rapidement la mort.

Les dosages ont été faits, pour le sodium, le potassium et le calcium par photométrie de flamme sur le plasma dilué non minéralisé (appareil Eippendorf), pour le magnésium et le phosphore total sur le plasma minéralisé par voie nitroperchlorique, le magnésium par la modification de LEwIS de la méthode au jaune de titane (rg6o), le phosphore par la méthode de Misson au phosphovanadomolybdate d'ammonium. Les protéines ont été dosées par réfractométrie (réfractomètre de ABBE). Dans chaque cas il a été récolté un échantillon d'herbe; l'étude de ces prélèvements fera l'objet d'une publication ultérieure.

L'exploitation des données a été faite pour chaque paramètre isolément au moyen d'une analyse de variance en regroupant les résultats en 5 classes de symptômes : 0 ; de 0,5 à I; de $I, 5$ à 2 ; de 2,5 à 3 ; de 3,5 à 5 . Les données ont été ensuite envisagées dans leur ensemble au moyen de l'équation de régression multiple qui relie les symptômes à la composition du plasma, après transformation éventuelle de chaque paramètre pour en rendre la distribution normale. 
TABLEAU 2

Principaux renseignements concernant les pâlurages de chaque exploitation

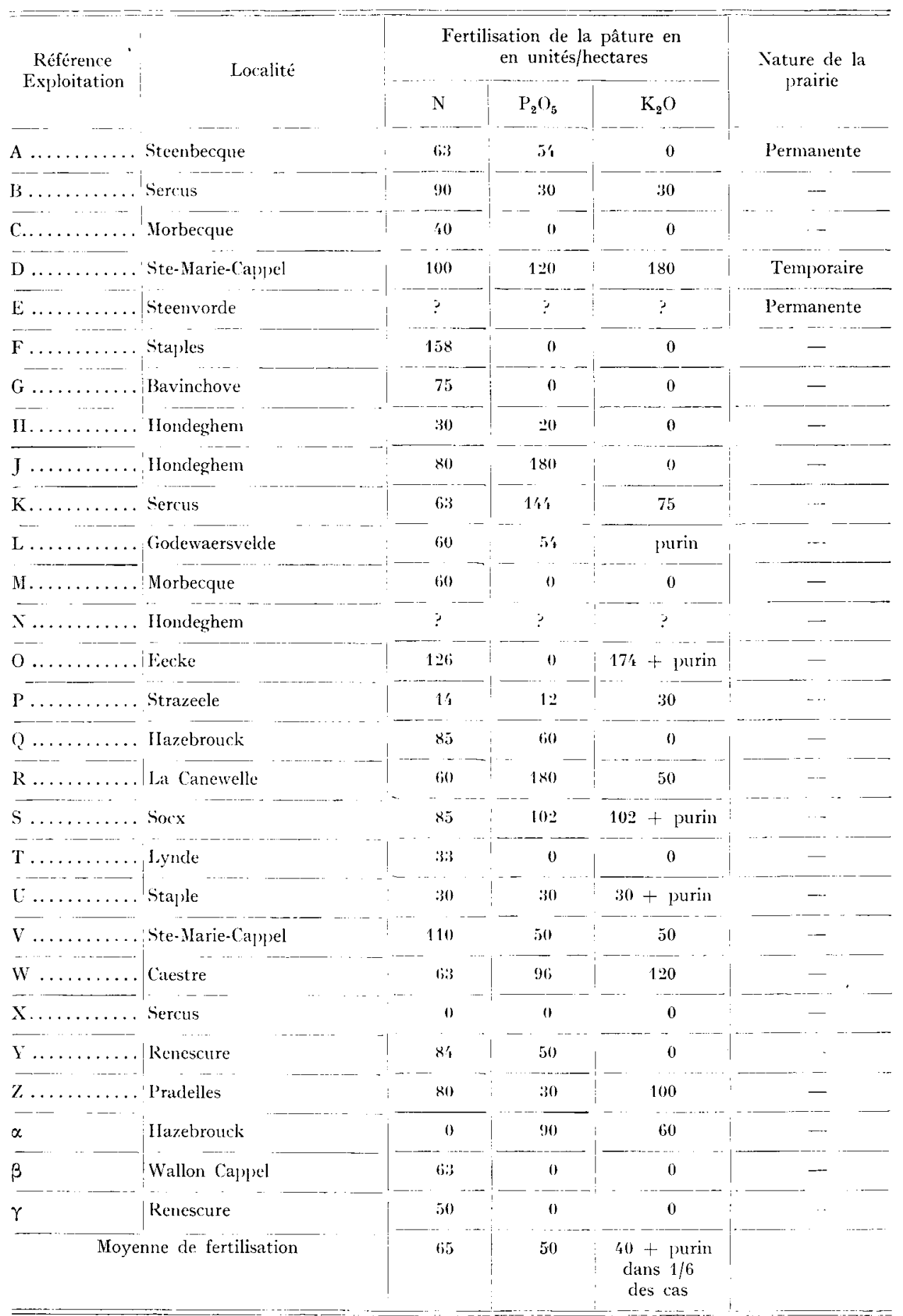

Annales de Zootechnie. - in62. 


\section{RÉSULTATS}

A) ÉVOI,UTION DE CHAQUE VARIABLE INDIVIDULILE EN YONCTION DES SYMPTÔMES

Le tableau I donne l'ensemble des résultats des analyses individuelles.

Io Variation $d u \mathrm{pH}$ plasmatique en fonction des symptômes (figure Ia)

Le pH plasmatique a augmenté mais de façon non significative avec l'aggravation des symptômes. La variance du $\mathrm{pH}$ est plus forte pour les symptômes graves, mais de façon non significative.

Il y a une corrélation positive significative $(r=+0,359, \mathrm{P}<0,05)$ entre le $\mathrm{pH}$ du plasma et la pression atmosphérique à la même heure, relevée à l'observatoire de Lille-Lesquin.

$2^{\circ}$ Variations du sodium plusmatique en fonction des symptômes (figure I $b$ )

Aucune variation significative en fonction des symptomes.

$3^{\circ}$ Variations du potassium plasmalique en fonction des symptômes (figure $1 \mathrm{c}$ )

Légère tendance, non significative, à l'augmentation avec l'aggravation des symptômes.

$4^{\circ}$ Variations du calcium plasmatique en fonction des symptômes (figure $\mathrm{I} d$ )

Abaissement progressif hautement significatif du calcium avec l'aggravation des symptômes. Lorsque les symptômes sont graves, les valeurs de Ca observées sont comparables à celles qu'on constate dans les hypocalcémies vitulaires.

$5^{\circ}$ Variations du phosphore total plasmatique en fonction des symptômes (figure I $e$ )

Aucune variation significative.

$6^{\circ}$ Variations du magnésium plasmatique en fonction des symptômes (figure I $f$ )

Il y a un abaissement progressif hautement significatif du magnésium avec 1'aggravation des symptômes. On constate aussi une relation entre le magnésium plamatique et le stade de la lactation au moment du prélèvement (figure 2; $\boldsymbol{r}=0,506, \mathrm{P}<0,01)$

La régression linéaire entre le magnésium plasmatique $(\mathrm{Mg})$ exprimé en mg pour Ioo $\mathrm{ml}$, et le stade de lactation (S. L.) exprimé en nombre de jours à compter du vêlage, est :

$$
\mathrm{Mg}=0,00508 \mathrm{SL}_{1}+0,482
$$

Il est à noter que l'on n'a pas observé de 'Tétanie à un stade de lactation inférieur à i 5 jours, et que la plupart des cas s'observent entre 20 et go jours de lactation.

$7^{0}$ Variations des protéines plasmatiques en tonction des symptômes (figure I $g$ )

Aucune variation significative. La dispersion plus grande des valeurs pour les animaux malades n'est pas significative. 

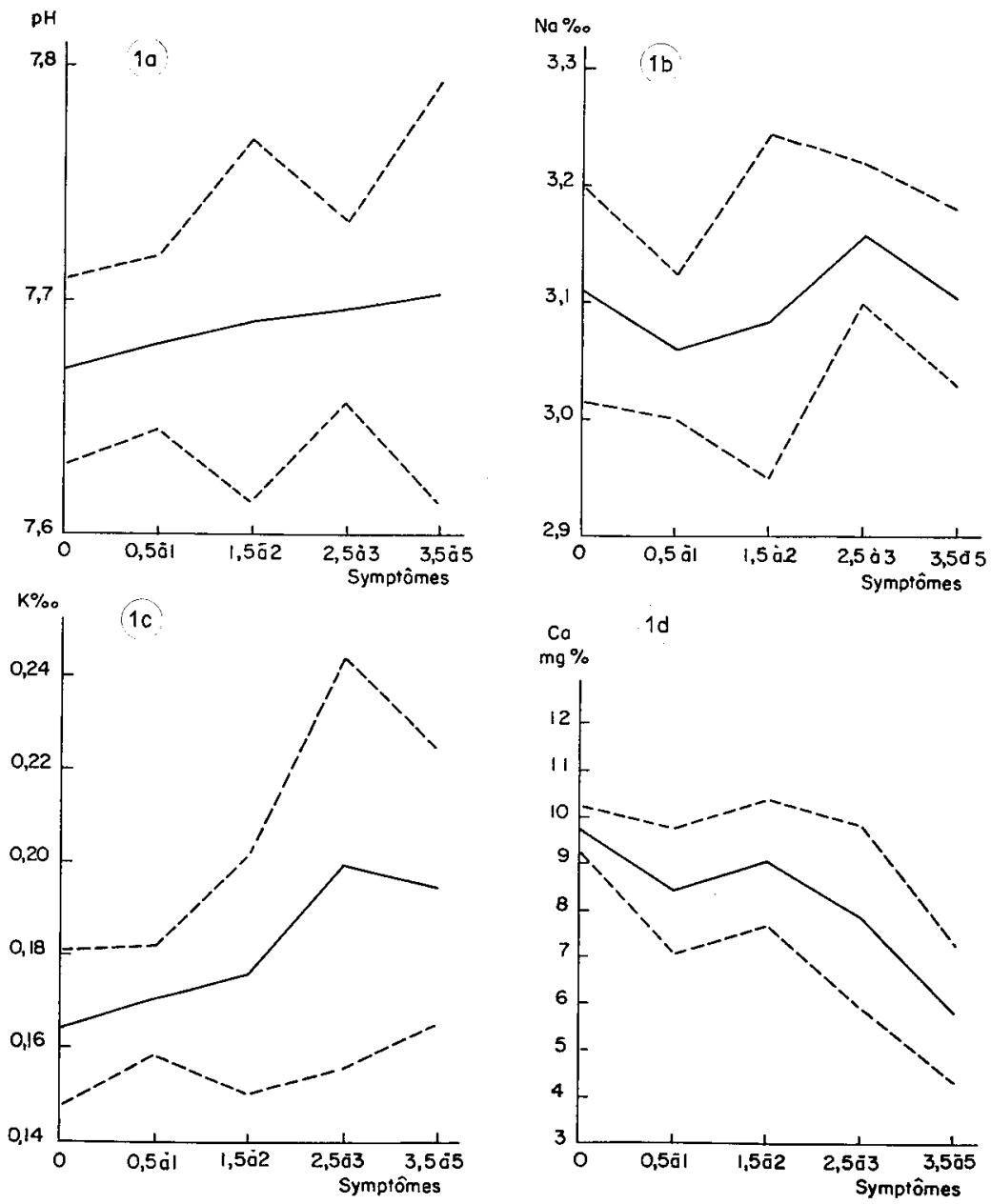

FIG. I. - a) Évolution du pH plasmatique en fonction des symplômes observés.

b) Sodium plasmatique.

c) Potassium plasmatique.

d) Calcium plasmatique.

La ligne pleine représente la valeur moyenne, les deux lignes interrompues représentent l'intervalle de confiance $\pm \frac{t \sigma}{\sqrt{\mathrm{N}}} \cdot$ 

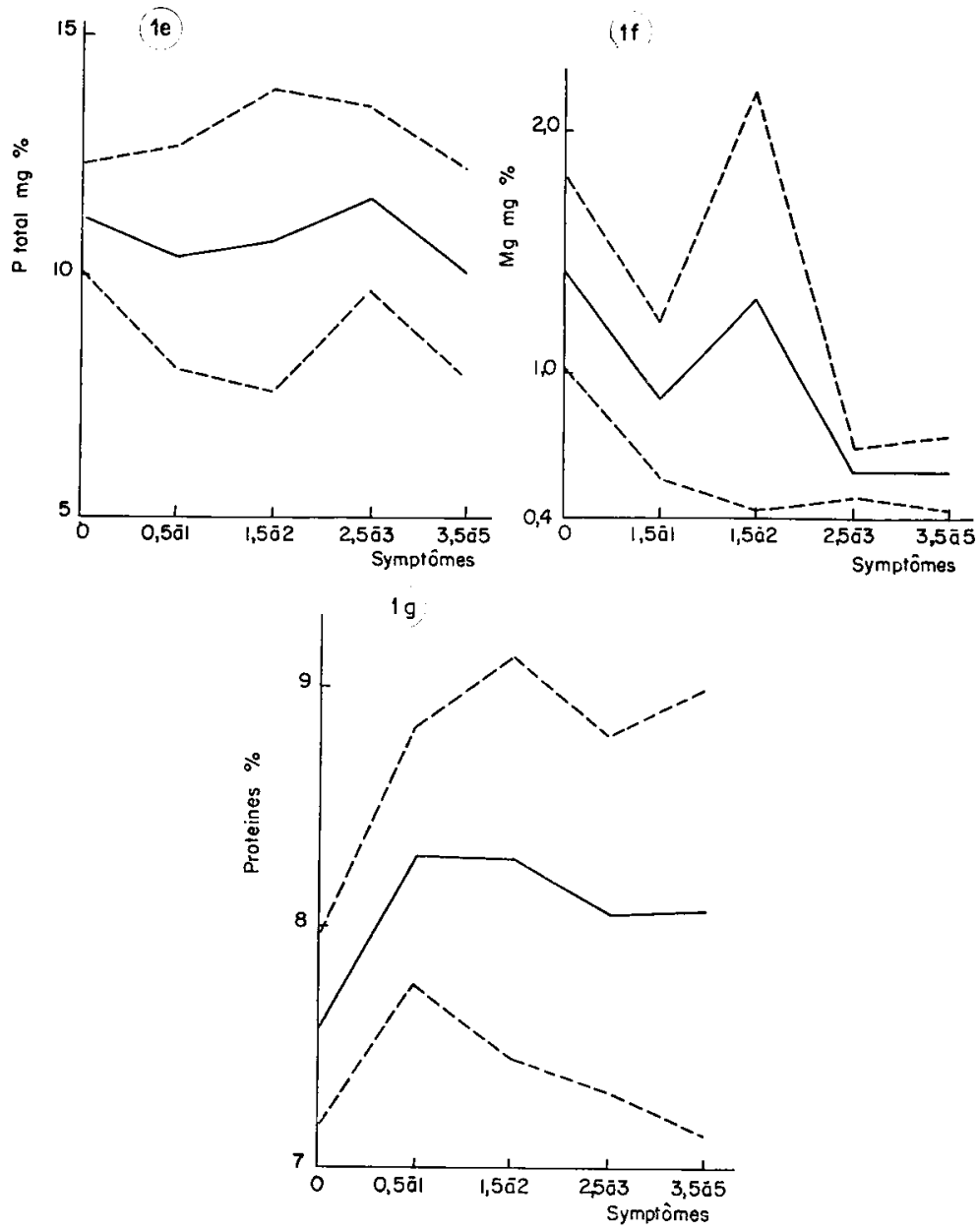

FIG. I. - e) Évolution du phosphore tolal plasmatique en fonction des symplóntes observés.

f) Magnésium plasmatique.

g) l'rotéines plasmatiques.

Ia ligne pleine représente la valeur moyenne, les deux lignes interrompues représentent l'intervalle de confiance $\pm \frac{t \sigma}{1 \times}$. 
B) RELATIONS GLOBALES ENTRE I,ES SYMPTÔMES ET LA COMPOSITION DU PLASMA

La régression linéaire entre les symptômes et la composition du plasma a été calculée sur ordinateur IBM 704, en tenant compte des transformations suivantes, de façon à normaliser les distributions :

Potentiel en ions hydrogène : sans transformation ; symbole $\mathrm{pH}$; unités habituelles de $\mathrm{pH}$.

Potassium : logarithme népérien ; symbole $\mathrm{K}$; exprimé en parties pour Io ooo.

Sodium : sans transformation; symbole $\mathrm{Na}$; exprimé en parties pour I ooo.

Calcium : logarithme népérien; symbole Ca ; exprimé en parties pour Ioo ooo (mg pour Ioo $\mathrm{ml}$ ).

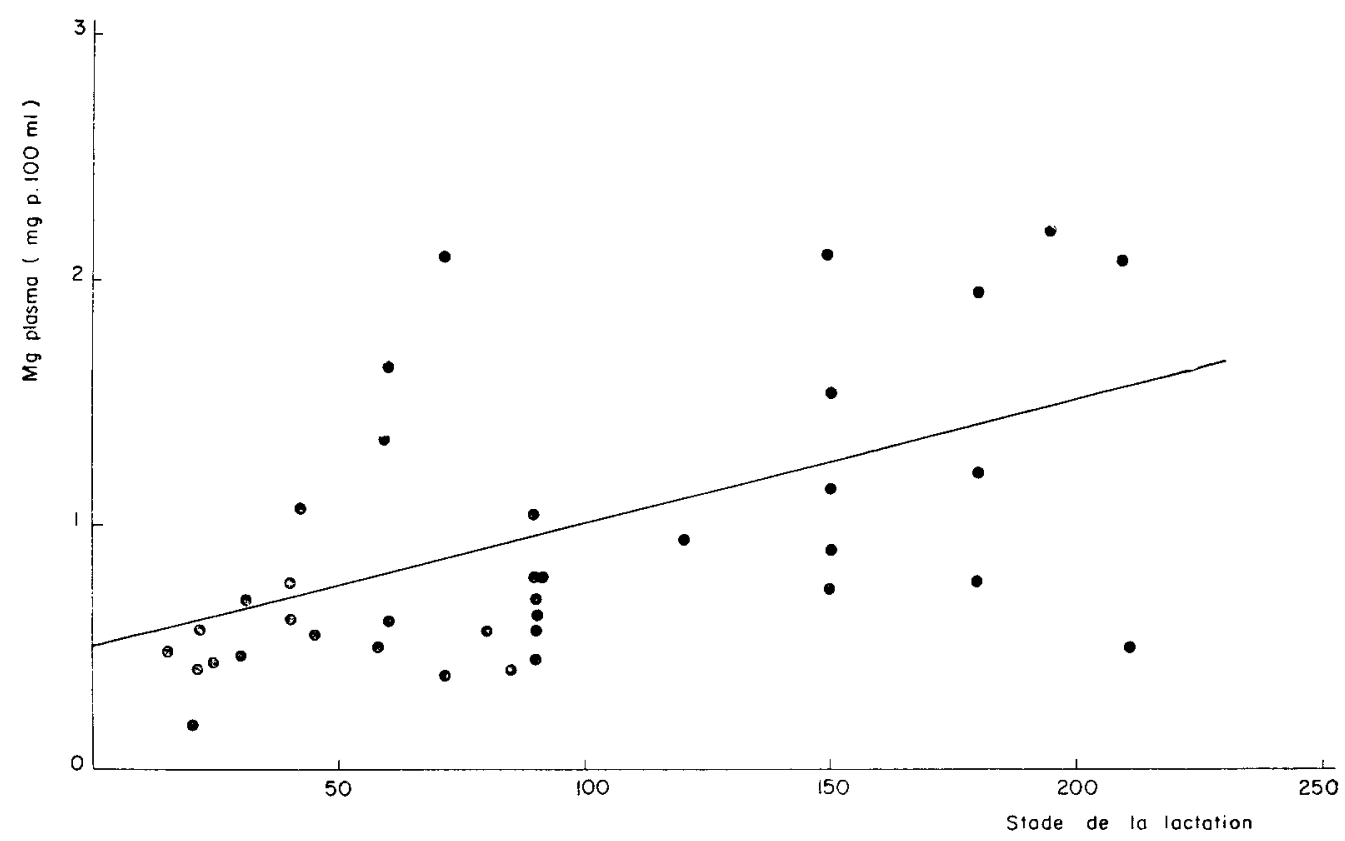

FIG. 2. - Relation entre le magnésium plasmatique el le stade de lactation au moment du prélèvement

Phosphore total : sans transformation; symbole $\mathrm{P}$; exprimé en parties pour IOO 000 ( $\mathrm{mg}$ pour IOO $\mathrm{ml}$ ).

Magnésium : logarithme népérien ; symbole $\mathrm{Mg}$; exprimé en parties par million.

Protéines : sans transformation ; symbole Pro ; exprimé en parties pour Ioo.

Ces différentes façons d'exprimer les résultats ont été choisies pour éviter l'emploi de logarithmes négatifs. On obtient la régression :

Symptômes $=\mathrm{S}=0,7 \mathrm{I} 6+\mathrm{I}, 40 \mathrm{I} \mathrm{pH}+0,3 \mathrm{I} 6 \mathrm{lg} \mathrm{n} \mathrm{K}-0,795 \mathrm{Na} \ldots$ .... - 3, Iro $\lg \mathrm{n} \mathrm{Ca}+0$, I $_{4} \mathrm{P}-0,600 \lg \mathrm{n} \mathrm{Mg}-0$, org Pro.

Dans ces conditions, la corrélation multiple entre $S$ et les composants du plasma est $\mathrm{R}=0,723$, c'est-à-dire que la fraction de la variance des symptômes explicable par les variations plasmatiques analysées est de $52 \mathrm{p}$. Iоo. 
Si on supprime les composants qui n'influencent pas significativement la régression, on parvient à la relation :

$$
\mathrm{S}=8,83-0,77 \lg \mathrm{n} \mathrm{Mg}-2,66 \lg \mathrm{n} \mathrm{Ca}
$$

ou, en transformant en logarithmes décimaux :

$$
\mathrm{S}=8,83-\mathrm{I}, 77 \mathrm{lg} \mathrm{Mg}-6, \mathrm{I} 2 \mathrm{lg} \mathrm{Ca} .
$$

La corrélation multiple est $\mathrm{R}=0,703$, c'est-à-dire que la fraction de la variance des symptômes qui se trouve contrôlée est de 49,4 p. Ioo. La corrélation est hautement significative (limite de $\mathrm{R}$ pour $\mathrm{P}<0, \mathrm{or}: 0,494$ ).

Le magnésium et le calcium sont donc les seuls critères plasmatiques envisagés qui puissent rendre compte d'une partie appréciable des symptômes.

\section{DISCUSSION}

A) VARIATIONS INDIVIDUELLES DES PARAMÈTRES PLASMATIQUES

Il convient de souligner quelques points particuliers, et de comparer les résultats obtenus avec les données bibliographiques.

$\mathrm{I}^{0}$ La relation positive entre $\mathrm{pH}$ dı plasma et pression atmosphérique a déjà été signalée chez l'homme et le chien par BERG et al. (1940). Elle pourrait être suscep-

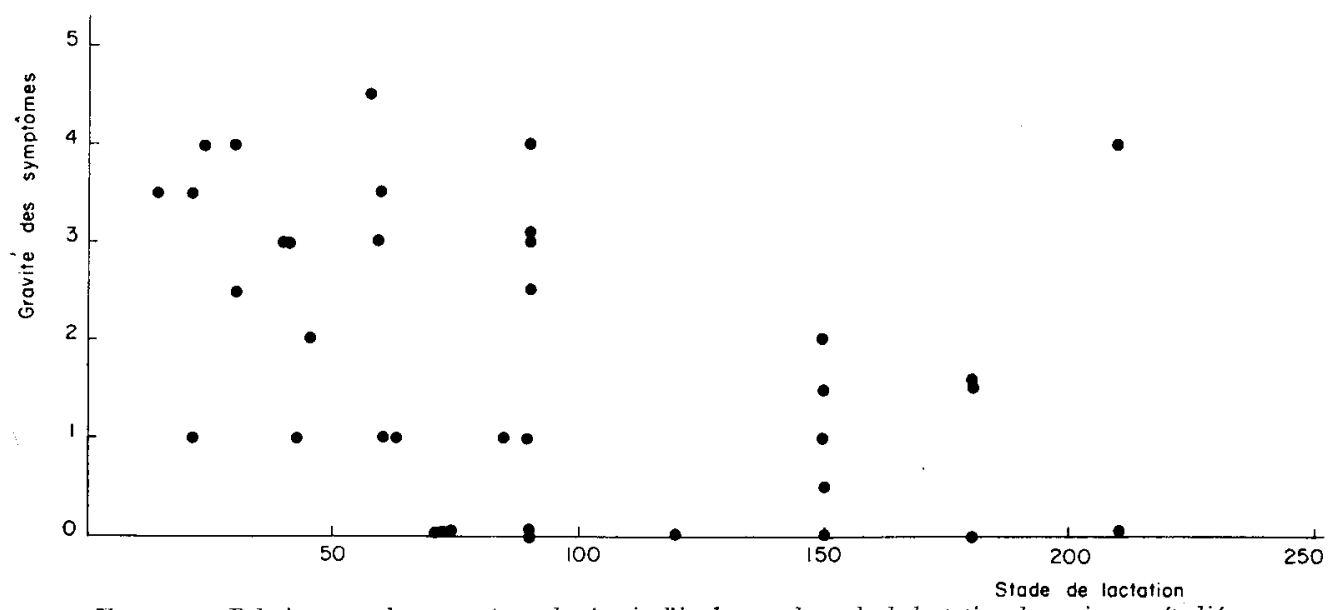

FIG. 3. - Relation entre les symptômes de télanie d'herbage et le stade de lactation des animaux étudiés

tible d'éclairer un fait bien connu des cliniciens, à savoir que les syndromes vitulaires et tétaniques sont particulièrement fréquents au moment de brusques changements de temps. En effet, l'ionisation des minéraux du plasma est en relation étroite avec le $\mathrm{pH}$ de celui-ci. Le $\mathrm{pH}$ n'a pas joué de rôle important dans le cadre de notre étude. mais celle-ci s'est déroulée pendant une période dépourvue de grandes perturbations atmosphériques. 
$2^{\circ}$ On n'a pas observé de relation entre la fréquence de la Tétanie d'herbage ou le magnésium plasmatique et la température. Là encore, il faut remarquer que les conditions climatiques ont été assez constantes pendant la période considérée; (température minimale moyenne $6,2^{\circ} \mathrm{C}$; température maximale moyenne $I 8,0^{\circ} \mathrm{C}$, pratiquement pas de précipitations).

$3^{\circ} \mathrm{La}$ corrélation positive entre le magnésium plasmatique et le stade de lactation n'est pas étonnante, puisqu'on a déjà démontré une corrélation négative entre la production laitière et le magnésium plasmatique (LADRAT, LARVOR et BROCHART, $\mathrm{r} 960$ ), due selon toute vraisemblance à ce que les vaches ont des besoins accrus en magnésium, et qu'il y a évidemment une relation négative entre le stade de lactation et la quantité de lait produite.

Le fait que la production laitière augmente généralement au cours dı premier mois de lactation n'est pas en contradiction avec cette hypothèse, puisque l'on n'a pas observé de cas de 'Tétanie d'herbage à moins de I5 jours de lactation et que la plupart des cas se sont produits entre 20 et 90 jours de lactation (figure 3 ).

$4^{\circ}$ Les données bibliographiques sur les variations de la composition sanguine dans la Tétanie ont été rassemblées dans le tableau 3, qui permet de faire un bilan d'ensemble de ces modifications. Le magnésium est considéré par la plupart des auteurs comme abaissé de façon très constante. Le calcium est considéré comme souvent abaissé, dans un pourcentage de cas très variable selon les auteurs; nous verrons plus loin que ces différences tiennent vraisemblablement à des différences de gravité dans le syndrome, ce que seule une étude quantitative (ou semi-quantitative) de la maladie pouvait mettre en évidence. I,e potassium est parfois considéré comme augmenté (diverses études portant sur jo cas en tout), parfois comme inchangé (études portant sur 76 cas en tout), parfois comme diminué (études portant sur 3 cas en tout). I a situation est analogue pour le phosphore minéral qui semble particulièrement variable. Le sodium est considéré comme inchangé. Il semble également que l'a\%ote résiduel soit très fréquemment élevé.

\section{B) ÉTUDE SIMULTANÍE DE LA VARIATION 1)E QUEI.QUES COMPOSANTS PIASMATIQUES}

Dans nos observations, le magnésium et le calcium plasmatiques ont seuls joué un rôle significatif. L'abaissement du calcium semble être un phénomène aussi important que celui du magnésium dans le déterminisme des crises ; il semble bien y avoir synergie entre les variations des deux minéraux.

Si on représente sur un graphique la gravité des symptômes en fonction de la teneur en $\mathrm{Mg}$ et Ca du plasma (figure 4) on s'aperçoit que le calcium, ne baisse presque jamais seul, tandis que le magnésium baisse fréquemment seul. Ceci suggère que le phénomène primaire est la baisse du magnésium tandis que la baisse du calcium vient se superposer plus tardivement en aggravarit fortement les symptômes. Cette interférence entre l'homéostasie du magnésium et celle du calcium n'est pas une simple vue de l'esprit, puisque R. H. SмI'TH (I96I) a montré que, chez les veaux de lait, la carence magnésienne entraîne une baisse du magnésium, puis du calcium plasmatique, en dépit d'apports en Ca et en vitamine I) satisfaisants. Chez ces veaux, une injection intraveineuse de $\mathrm{Mg}$ ou un supplément alimentaire de $\mathrm{Mg}$ entraînent immédiatement la remontée du Mg et dı Ca sanguins. L’importance symptomatologique de la baisse 
TABLEAU 3

Récapitulation des données bibliographiques sur la composition du sérum ou du plasma dans la Tétanie d'herbage. Dans les colonnes $\mathrm{Mg}$ et $\mathrm{Ca}$, on a porté dans certains cas, entre parenthèses, la proportion des cas dans lesquels ces deux paramètres étaient abaissés. Ixemple : (16/20) dans la colonne Ca signifie que pour les auteurs considerés, il y anait i 6 hypocalcimies sur les 20 cas examinés.

\begin{tabular}{|c|c|c|c|c|c|c|c|c|}
\hline Auteurs & & $\left|\begin{array}{c}\text { Nombre } \\
\text { de cas }\end{array}\right|$ & $\mathrm{Hg}$ & $\mathrm{Ca}$ & $\mathbf{K}$ & $\mathrm{Na}$ & $\begin{array}{l}\text { P mi- } \\
\text { néral }\end{array}$ & Divers \\
\hline $\begin{array}{l}\text { Sjollema et SeEkles.... } \\
\text { Sjoldema et SEekles... }\end{array}$ & $\begin{array}{ll}1930 & \vdots \\
1932 & \vdots\end{array}$ & $?$ & - & $\cdots$ & $=$ & $=$ & & \\
\hline DRYERRE. $\ldots \ldots \ldots \ldots$ & 1932 & & - & $=\overline{\mathrm{ou}}-$ & $=$ ou $t$ & $=$ oul & & \\
\hline BLAKEMORE et STEWART & 1933 & & - & & & & & \\
\hline$\overline{\text { HopKIRK et }}$ al.......... & 1933 & 35 & - & - & $=$ & $=$ & \pm & \\
\hline ALLCROFT et GREEN.. & $193 / 1$ & & - & - & & & & \\
\hline METZGER & 1936 & 1 & - & 一 & & & - & $\begin{array}{l}\text { (ilucose }=; \\
N \text { non protéique } \\
=\text {; Acétone } 0 .\end{array}$ \\
\hline Nolan et Hull $\ldots \ldots$ & $19^{\prime} 1$ & 12 & - & - & & & - & $\begin{array}{l}\text { Glucose parfois }+ \text {; } \\
\mathrm{N} \text { non Protéique }=\text {. }\end{array}$ \\
\hline MuTH et IAAAg.......... & 19,5 & 1 & - & $(1 / 1)$ & & & & \\
\hline LLCROFT ........... & $\left(\begin{array}{c}19 \% \\
(a \text { et } b)\end{array}\right.$ & $\begin{array}{l}\text { Nom- } \\
\text { breux }\end{array}$ & $\ldots$ & $\left(\begin{array}{cc}76 & 0\end{array}\right)$ & & & & \\
\hline HARBAUGH et DENNIS.... & 19,7 & 6 & & - & + & & & \\
\hline UDALL. & 1917 & 3 & - & $(3 /:)$ & & & $=$ & $\mathrm{Cl}=$ \\
\hline Sims et Crookshank .... & 1953 & 17 & - & - & + & & $\therefore-$ & \\
\hline INGLIS et $a l, \ldots \ldots \ldots$ & $19 \overline{5}$ & 8 & - & & & & & \\
\hline Van Koltsveld ... & 1955 & 8 & & & & & & $\begin{array}{l}\mathrm{SO}_{4}+(\text { cas de pré. } \\
\text { tétanie). }\end{array}$ \\
\hline $\begin{array}{l}\text { SINGER et } a l . \ldots \ldots \ldots \\
\text { SINGER et } a l . \ldots \ldots \\
\text { GRAINGER et } a l . \ldots \ldots \ldots\end{array}$ & $\left.\begin{array}{l}1956 \\
1958 \\
1959\end{array}\right)$ & 27 & $(\overline{5 / 27})$ & 一 & + & $=$ & + & $\begin{array}{l}\mathrm{Cu} ; \text { bilirubinémie } \\
\mathrm{N} \text { non protéique } \\
+; \mathrm{Cl}=\end{array}$ \\
\hline Evans et Pinluipson .... & 1957 & 1 & & - & - & $=$ & & $\mathrm{H}_{2} \mathrm{O}-$ \\
\hline MrTH et al. & 1958 & 2 & -- & $=$ & $=$ & & & $\begin{array}{l}\text { Sucres }=; \\
\text { Cétones }=\end{array}$ \\
\hline $\begin{array}{l}\text { Mersion et Custer } \ldots \ldots \\
\text { Custer } \ldots \ldots \ldots \ldots \ldots\end{array}$ & $\begin{array}{l}1958 \\
1959\end{array}$ & 20 & - & $16 / 20$ & & & & $\begin{array}{l}\text { Tétanie } \\
\text { d'étable }\end{array}$ \\
\hline$\ddot{M}_{\Lambda R S I L K K}-\ldots \ldots \ldots \ldots \ldots$ & $195 \overline{9}$ & 1 & - & $\cdots$ & & & & Glucose $=$ \\
\hline HORYATH $\ldots \ldots \ldots \ldots$ & 1959 & 2 & $\cdots$ & $=-$ & $=$ & & & - \\
\hline LADRAT et $a l, \ldots \ldots \ldots$ & 1959 & 8 & - & $(1 / x)$ & $=\mathrm{ou}$ & $=$ & $=$ & $\begin{array}{l}\text { l total }=: \\
\mathrm{Cl}=; \mathrm{H}_{2} \mathrm{O}-.\end{array}$ \\
\hline Hugites et CoRnelits . . & 1960 & 2 & - & - & - & & & Protéines $=$ \\
\hline Charton ... & 1960 & $\div 2$ & $(\overline{1 s / 2 \cdot 2)}$ & $(9 / 2,2)$ & & & \pm & $\begin{array}{l}N \text { Résiduel + ; } \\
N \text { non protéique } \\
+. \text { urée }+ \text {; }\end{array}$ \\
\hline $\begin{array}{l}\text { STORRY } \cdots \cdots \cdots \cdots \\
\text { STORRY } \ldots \ldots \ldots \ldots \cdots\end{array}$ & $\begin{array}{l}1960 \\
1961\end{array} \mid$ & $\begin{array}{ll}\cdots \\
5\end{array}$ & - & - & & & & \\
\hline Présente étude ..... & 1962 & 31. & - & $\overline{(15 / 31)}$ & $=$ & $=$ & & $\left\{\begin{array}{l}\mathbf{P} \text { total }=; \\
\mathrm{pH}=; \\
\text { Protéines }=\end{array}\right.$ \\
\hline
\end{tabular}


du calcium plasmatique ne doit donc pas faire oublier que la cause première est un trouble du métabolisme du magnésium.

Une autre question importante peut-être posée : les variations du magnésium et du calcium plasmatiques suffisent-elles à expliquer l'apparition des symptômes, ou faut-il faire intervenir un autre facteur ? On a vu que les variations de $\mathrm{Mg}$ et $\mathrm{Ca}$

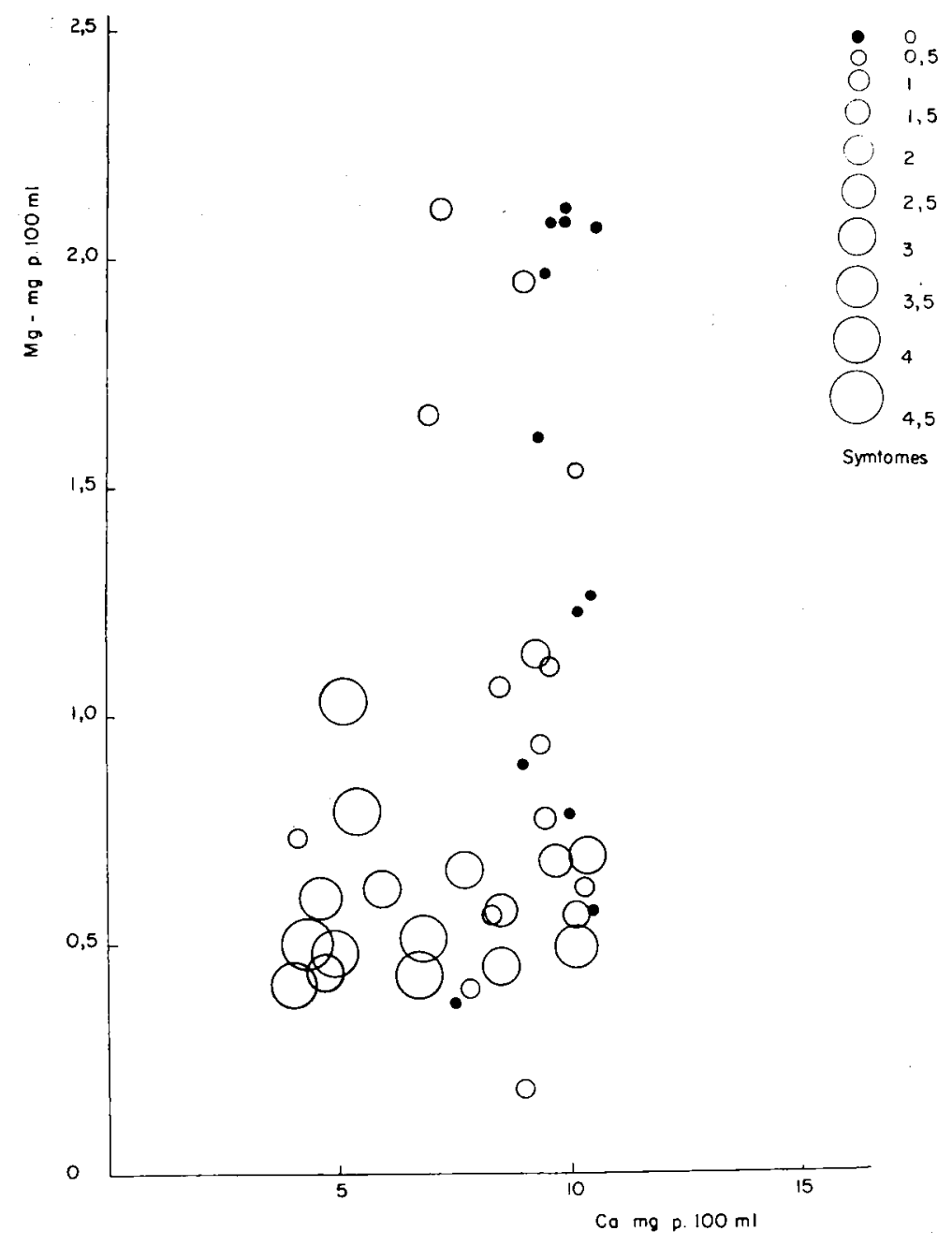

FIG. 4. - Gravité des symptômes en tonction du magnésium et du calcium plasmatiques

étaient susceptibles de rendre compte d'approximativement $50 \mathrm{p}$. Ioo de la variance des symptômes ; les $50 \mathrm{p}$. Ioo restant pourraient être attribués, $a$ ) soit aux erreurs dans l'appréciation arbitraire de la gravité de la Tétanie, $b$ ) soit aux erreurs dans l'estimation de $\mathrm{Mg}$ et $\mathrm{Ca}$, que l'on peut elles mêmes décomposer en erreurs analytiques et en erreurs sur la représentativité du prélèvement $c$ ) soit aux différences individuelles des animaux dans la réaction à une même modification humorale, $d$ ) soit 
enfin au fait qu'un autre élément que $\mathrm{Ca}$ et $\mathrm{Mg}$ puisse intervenir dans le déterminisme des symptômes.

Les erreurs dans l'appréciation subjective de la gravité des symptômes ont certainement été assez importantes ; on peut également supposer que la représentativité de l'échantillon n'a pas toujo'urs été rigoureuse, notamment lorsque le prélèvement a été fait dans la période de décroissance d'une crise tétanique ; RAY (I942) et Thomas (I959) ont en effet montré que chez le veau hypomagnésiémique, il y a un accroissement du Mg plasmatique pendant et juste après les convulsions ; enfin bien que l'on connaisse mal les variations individuelles de la sensibilité aux hypomagnésiémies et aux hypocalcémies, il est à présumer qu'elles peuvent être importantes.

Il semble que ces causes de variation prises ensemble, et principalement les erreurs dues aux prélèvements tardifs, puissent faire que, de temps à autre, on observe un cas de Tétanie d'herbage avec un magnésium sanguin relativement élevé.

Il est donc vraisemblable que les variations de $\mathrm{Mg}$ et Ca suffisent à expliquer les symptômes tétaniques sans faire intervenir d'autre facteur essentiel, sans toutefois qu'on puisse être formel à ce sujet.

Reçu en mai 1962.

\section{REMERCIEMENTS}

Nous tenons à remercier ici Messieurs les docteurs vétérinaires M. Comys et B. Hauwen, de Hazebrouck, M. CorNetTe, de Bergues et V. Delarte, de Cassel, grâce à l'obligeance et à la compétence desquels cette étude a pu être effectuée.

\section{SUMMARY}

THE RELATIONSHIP BETWEEN THE COMPOSITION OF BLOOD PLASMA AND TIE SYMPTOMS OF GRASS TETANY IN CATTLE

The relationship between some of the blood plasma components (calcium, total phosphorus, magnesium, sodium, potassium, $\mathrm{pH}$ and proteins) and the symptoms of Grass Tetany have been studied in 43 samples of plasma taken from 12 normal cows and from 31 cows suffering from different stages of the disease. Calcium, potassium and sodium were estimated by flame photometry; magnesium by Lewis' modification of the Titan Yellow method; phosphorus by the phosphovanadomolybdate method; and the proteins by refractometry. The symptoms were classified on arbitrary o to 5 scale according to subjective observation.

The regression equation between symptoms and plasma constituents had been calculated : only the reduction of plasma magnesium and calcium could explain a significant fraction of the variation in the symptoms. The multiple correlation coefficient connecting plasma magnesium and calcium with Grass Tetany symptoms was $R=0.703$ (the limit of $R$ being 0.494 for $P<0.01$ ). When taking into consideration the many sources of error, it seemed possible that the variations in plasma magnesium and calcium might account for all the symptoms observed.

Positive relationship were observed between plasma $\mathrm{pH}$ and atmospheric pressure $(r=+0.359$, $\mathrm{P}<0.05)$ and between plasma magnesium and the number of days aftercalving $(r=+0.506,<\mathrm{P} 0.0 \mathrm{I})$.

\section{RÉFÉRENCES BIBLIOGRAPHIQUES}

Allcroft W. M., 1947 a. Observations on some metabolic disorders of cows, as evidenced by chemical analysis of samples of blood from clinical cases. I) Condition of occurence. Vet. J., 103, 2-1 r.

ALICROFT W. M., I 947 b. Observations on some metabolic disorders of cows as evidenced by chemical analysis of samples of blood from clinical cases. 2) Some aspects of blood chemistry. Vet. J., 103, 30-47. 
Allcroft W. M., Green H. H., 1934. Blood calcium and magnesium of the cow in health and disease. Biochem. J., 28, 2220-2228.

Berg M., Mayne A., Petersen W. F., i940. Variability of blood pH and its association with meteorological factors. Amer. J. Physiol., 130, $g^{-21}$.

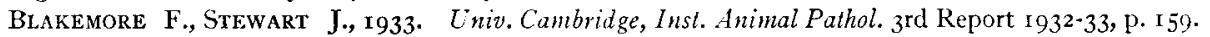

Charton A., 1960. Perturbations de la calcémie et de la magnésiémie dans les névroses d'origine nutritionnelle chez la vache laitière. Rev. Pathol. Génér. Physiol. Clin., 60, 1465-1478.

Custer F. D., r959. Tetany in cattle on winter rations. In : Magnesium in Agriculture, Symposium at West Virginia Univ., 3 et 4 Sept. 1959, p. r 59-162.

DRYERRE. H., 1932. Lactation tetany. Vet. Rec., 12, I I63-I 65.

Evins J. V., Pinllipson A. T., 1957. Electrolyte concentration in the erythrocytes of the goat and ox. J. Physiol., 135, 87-96.

Grainger R. B., Singer R. H., Baker R. II., Strovd J. W., I959. Investigations on a complicated tetany syndrome in ruminants of Kentucky. In : Magnesium in Agriculture, Symposium at West Virginia Uiniv., 3 et 4 sept. 1959 , p. 19x-195.

Harbaugh F. G., Dennis J., i947. The blood potassium and calcium levels of cattle grazing wheat. Amer. J. Vet. Res., 8, 396-399.

Hopkirk C.S. M., Marsual. D., Blake T. A., 1933. Grass tetaliy of dairy cows. Vel. Rec, 13, 355-362.

Horvarir D. J., 1959. So-called a Grass Tetany " in West Virginia : Survey and laboratory findings. In : Magnesium in Agriculture, Symposium at West Virginia Univ. 3 et 4 sept. 1959, p. 197-21 7 .

Hughes J. P., Cornelius C. E., 196o. An outbreak of grass tetany in lactating beef cattle. Cornell Vet 50, 26-33.

INglis J.S.S.. Weipers M., Marr A., I954. Some observations on bovine hypomagnesaemia. Vet. Rec., 66, 353-354.

Ladrat J., Laryor P., Brochart M., I959. Recherches sur quelques cas de Tétanie d'herbage. Rev. Méd. Vét., 135, 903-936.

Larvor P., Brochart M., et Théret M., r96r. Enquête sur la Fièvre Vitulaire et la Tétanie d'herbage des bovins en France. Économie et Médecine Animales, 2, 5-38.

LEwis W. I. P., 1960. A micro-method for the estimation of magnesium. J. Med. Laboratory Technol., 17, 32-35.

MARsiak R. R., r959. The role of magnesium in the tetany syndromes of cattle. In : Magnesium and Agriculture, Symposium at West Virginia Univ., 3 et 4 sept. 1959, p. 169-1 78.

Mershon M. M., Custer F. D., r958. Tetany in cattle on winter rations. Part I A clinical report. J. Amer. Vet. Med. Ass., 132, 396-400.

Metzger H. J., I936. A case of tetany with hypomagnesaemia in a dairy cow. Cornell Vet., 26, 353-356.

Mutil O. H., HAAG J. R., 1945. A disease of Oregon cattle associated with hypomagnesamia and hypocalcemia. North Amer. Vet., 26, 216-2 I9.

Nolan A. F., Hulc F. E., i94I. Grass tetany in cattle. Amer. J. Vet. Res., 2, 41-45.

RAY S. N., 1942. Hypomagnesaemia in heifer calves. Indian J. Vet. Sci. Anim. Husb., 12, 204-2 2.

Sims F. H., Crookshank H. R., 1953. Serum values in "wheat pasture poisoning" cases. J. Anim. Sci., 12, $954-955$.

Singer R. G., Grainger R. B., Powell C., i956. Grass tetany. Kentucky Agric. Exper. Sta., 69th Annual Repi., P. 75.

Singer R. H., Grainger R. B., BAKer R. H., 195\%. Investigations on a complicated Grass Tetany syndrome in ruminants of Kentucky. I. Preliminary observations. Kentucky Agric. Exper. Sta., Bull. 658, 5 pp.

Sjollema B., I930. On the nature and therapy of grass staggers. Vet. Rec., 10, 425-430 et 450-453.

Sjollema B., Seekles L., 1930. Ueber störungen des Mineralenregulation Mechanismus bei Krankheiten des Rindes. Biochem. Zischr., 229, 358.

Surif R. If., 196r. Importance of magnesium in the control of plasma calcium in the calf. Nature, 191, $18 \mathrm{I}+\mathrm{I} 82$.

Smyth P. J., Conway A., Walsh M. J., I958. The influence of different fertiliser treatments on the hypomagnesaemia proneness of a rye grass sward. Vet, Rec., 70, 846-849.

STORRY J. E., 1960. Studies on calcium and magnesium in the ruminant in relation to the aetiology of grass tetany. Thèse Sciences. Aberdeen, $50 \mathrm{pp}$.

STORRY J. E., 196r. Changes in blood constituent which occur in dairy cattle transferred to spring pastures. Res. Veter. Sci., 2, 272-284.

Thomas J. W., 1959. Magnesium nutrition of the calf. In : Magnesium and Agriculture. Symposium at West Virginia Univ., 3 et 4 sept. I959, p. I 3 I-I 50.

USDALL R. H., I947. Low blood magnesium and associated tetany occuring in cattle in the winter. Cornell Vet., 37, 3:4-324.

VAN KoEtsveld E. E., 1955. Welke betekenis moeten wij toekennen aan zwavel voor plant en dier, in het bijzonder voor het rund ? Tijdschr. Diergeneesk., 80, 525-550. 